\title{
2D and 3D Thermal Flow Sensor Modelling - A Comparative Analysis
}

\author{
Ethan L.W. Gardner ${ }^{*}$, Andrea De Luca ${ }^{*}$, ,* Florin Udrea*, ** \\ "University of Cambridge, Cambridge, United Kingdom \\ ${ }^{* *}$ Flusso Ltd, Cambridge, United Kingdom \\ elwg2@cam.ac.uk ad597@cam.ac.uk fu10000@cam.ac.uk
}

\begin{abstract}
In this paper, 2-Dimensional and 3Dimensional numerical models of a thermal flow sensor are discussed and compared against one another whilst being validated with experimental results. The models involve and couple three physics domains: electric, computational fluid dynamics and heat transfer. A comparative analysis has been performed focusing on the relative merits. Despite being less accurate, it is shown that $2 D$ simulations can be used to portray device behavior whilst minimizing required computational resources whereas 3D models are needed to attain more accurate quantitative data. This ultimately provides the knowledge for objective driven modelling decisions.
\end{abstract}

Keywords- Numerical Modelling; Flow Sensor; MEMS; CMOS.

\section{Introduction}

Due to the no-slip condition, when a fluid is flowing in contact with a solid boundary (in our case the surface of the flow sensor) there is a shear stress exerted due to the viscosity of the fluid. An additional result of the fluid/surface interaction is the creation of a velocity boundary layer and a thermal boundary layer. Analytical approaches for the description of these phenomena are not viable, so usually numerical approaches are employed [1-4] and can indeed be used to obtain useful information for an appropriate design of the flow sensor itself. The problem is to understand which assumptions (e.g. 2D or 3D geometry, single-physics or multiphysics, etc.) can be made in order to minimize the computational load whilst concurrently maintaining accurate results.

In this paper, a numerical model has been created based upon a diode-based MEMS thermal wall shear stress sensor fabricated in SOI CMOS technology. It utilizes and couples three physics domains: electric, thermal and fluid mechanics. This model is applied to 2-Dimensional (2D) and 3-Dimensional (3D) geometries. The results are validated against experimental data, as well as comparing them against each other, allowing their accuracy and practicality to be discussed.

\section{Sensor structure}

The flow sensor cross-section is shown in Fig. 1. Tungsten is used as metal layer for the five resistors with dimensions $2 \mu \mathrm{m} \times 400 \mu \mathrm{m} \times 0.3$ $\mu \mathrm{m}$ (Fig. 2). In this study only the central wire is biased with a constant current to act as a heat source. Underneath each resistor there is a temperature sensing diode, fabricated in the thin silicon layer. When forward biased with $10 \mu \mathrm{A}$, the voltage drop $(V d)$ across each diode can be correlated to temperature as follows: $V d=$ $-0.00157 T+0.76$. This sensing structure provides the ability to run the sensor in anemometric or calorimetric mode.

The chip was fabricated with standard SOI CMOS technology, benefiting from a deep reactive ion etching at the back surface to dramatically reduce the thermal conductivity. A detailed description of the diode-based thermal flow sensor can be found here [5].

\section{Numerical Simulation}

\section{A. Multi-Physics Model}

The numerical model is implemented via COMSOL Multiphysics. Three separate physics domains are coupled together to simulate the device behaviour: (a) a biasing current is used to

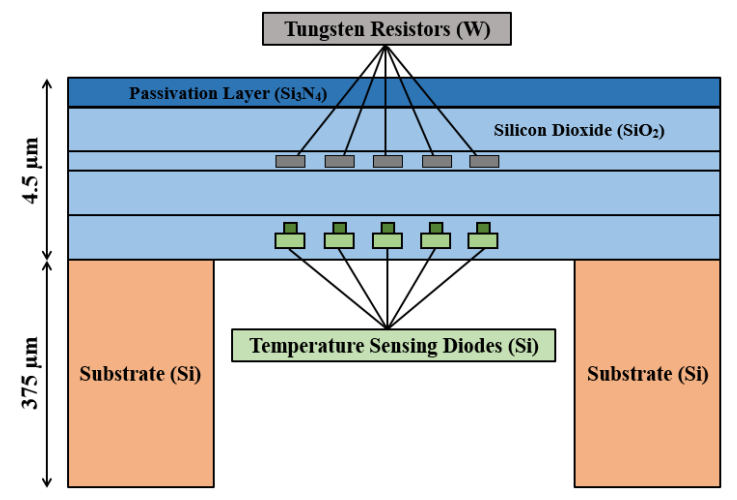

Fig. 1. Cross-section of sensor, fabricated in SOI CMOS MEMS technology (not to scale). 


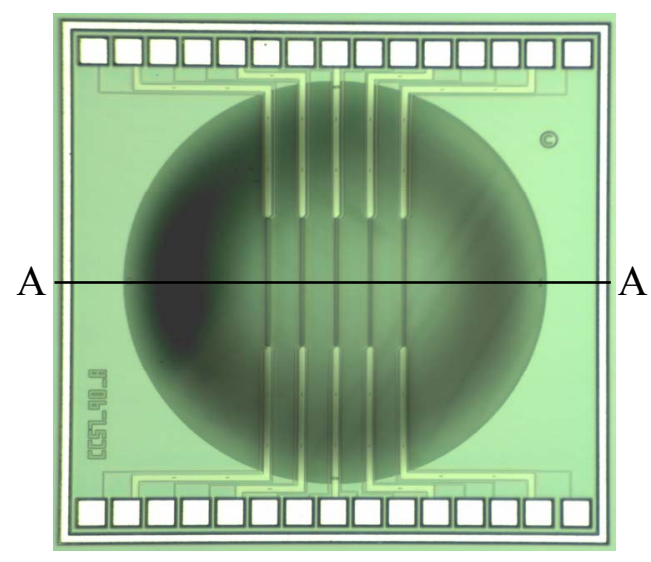

Fig. 2. Optical micrograph of the fabricated thermoelectronic flow sensor (size: $1.6 \mathrm{~mm}$ x $1.6 \mathrm{~mm}$ ).

locally heat up the resistor (Joule Heating), (b) there is heat transfer from the resistor through the membrane towards the substrate (via conduction) and through the fluid above and below the membrane (via conduction and convection) and (c) laminar flow is introduced through the adjacent fluid, affecting the forced convection from the heated membrane area into the fluid. More details on the equations and boundary conditions can be found here [4]. It should be noted that most material properties are highly temperature dependent; therefore interpolative lookup tables were defined for specific heat, thermal expansion, heat transfer coefficients, densities, and viscosities and used in the models. The high temperature generated by the heater results in significant natural convection, which plays a dominant role in stagnant flow conditions. The Archimedes number, $\mathrm{Ar}=\mathrm{Gr} /$ $R e^{2}$, parameterizes the relative strength of free and forced convection, $G r$ is the Grashof number, a dimensionless parameter which approximates the ratio of buoyancy force to viscous force to correlate thermally induced natural convection, and $R e$ is the Reynolds number, a dimensionless parameter of the ratio between inertial and viscous forces. Due to the model considering highly laminar flow, the Archimedes number warrants that natural convection cannot be omitted ( $A r$ is not $<<1)$, and is therefore considered for all flow rates.

\section{B. 2-Dimensional Model}

Fig. 3 shows the 2D model created, located at the A-A slice shown in Fig. 2. The model contains a section underneath the membrane with stagnant air where conduction and natural convection equations are solved. Over the sensor

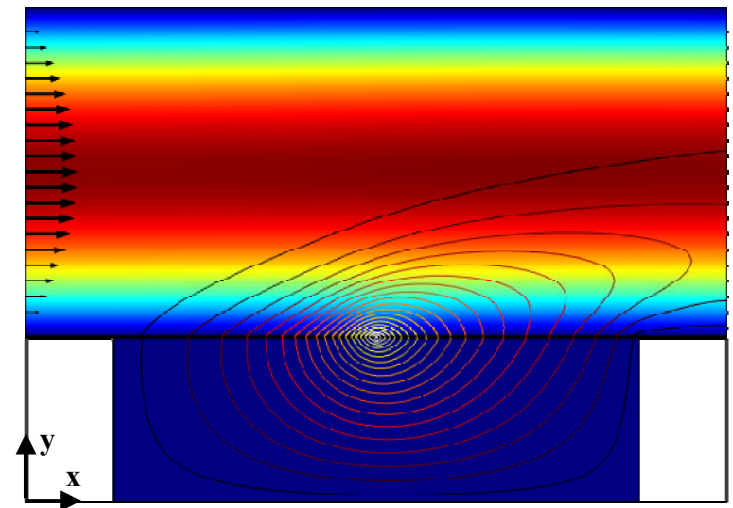

Fig. 3. An example of $2 \mathrm{D}$ simulation results with air flowing above the sensor. A thermal contour plot as well as velocity colour mapping are presented along with arrows indicating the flow velocity profile.

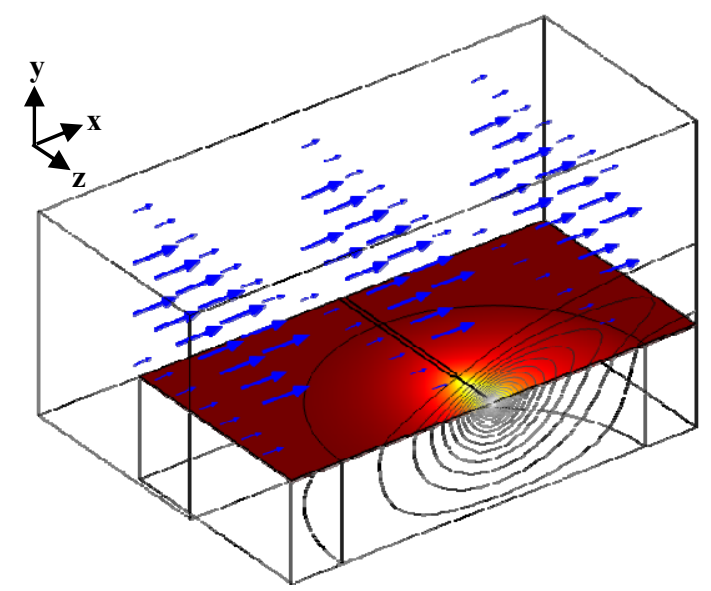

Fig. 4. An example of 3D simulation results with air flowing above the sensor. A thermal contour plot as well as surface temperature distributions are presented along with arrows indicating the flow velocity profile.

is another region of air that represents the channel. In this region conduction, natural and forced convection equations are solved. All this is coupled with the Joule heating generated within the membrane, where conduction equations are also solved.

\section{3-Dimensional Model}

Fig. 4 shows the full 3D model of the sensor. Similarly to the 2D model, underneath the circular membrane is a volume of stagnant air and above it an air channel. The two lateral channel walls were defined as outflow and so do not exhibit the no-slip condition.

\section{Discussion}

Firstly, it is paramount that the biasing current and power dissipation correlate accurately to the temperature change of the heater. Fig. 5 shows 
the temperature output of the heater with respect to the applied power. The 3D simulation perfectly matches the experimental data, leading to the conclusion that this simulation is accurately depicting Joule Heating. Whilst showing the same trend, the $2 \mathrm{D}$ simulation is predicting temperatures greater than is seen experimentally, with a sensitivity error of $16 \%$ $\left(3.4{ }^{\circ} \mathrm{C} / \mathrm{mW}\right)$. This over-estimation of the heater temperature can be explained by some of the simplifications in the geometry. Most significantly, the 2D geometry does not incorporate the length of the tungsten heater and the tungsten tracks leading to the membrane edge. This cannot be neglected because there is a large amount of heat transfer via conduction transporting heat away from the heater through this metal track and hence reducing the temperature. In addition to this, the 2D simulation only considers the thermal resistances along $x$ and $y$ directions, meaning the heat dissipation in the $z$-direction through conduction and convection is being neglected. In opposition to this, the 2D simulation is unable to model the membrane as circular, but instead assumes a square membrane which results in larger thermal resistance. However, this factor has less effect in comparison with the previous points.

Knowing that a 2D simulation over-estimates heater temperature, this temperature will henceforth be matched to the experimental value to compensate for the 2D inaccuracy and provide a valid comparison to the $3 \mathrm{D}$ model in terms of on-membrane temperature profile distribution. Fig. 6 shows the temperature profile parallel to the direction of flow for stagnant conditions $\left(\tau_{w}=\right.$ $0 \mathrm{~Pa})$ and laminar flow $\left(\tau_{w}=1 \mathrm{~Pa}\right)$. Again, the 3D simulation accurately matches the experimental data with all errors being under 5\% which validates that the multi-physics coupling between the heating of the resistor and the heat transfer through the remaining structure is valid. The addition of forced convection via moving air was validated by measuring temperatures for a wall shear stress up to $1 \mathrm{~Pa}$, again with errors all below $5 \%$, proving that the $3 \mathrm{D}$ simulation accurately models and couples all three physics throughout the geometry. After temperature compensation, the 2D simulation does not display the same temperature profile, with a general over-estimation of the temperature at any point. This behavior can be explained be the incorrectly assumed thermal resistance in the $z$ -

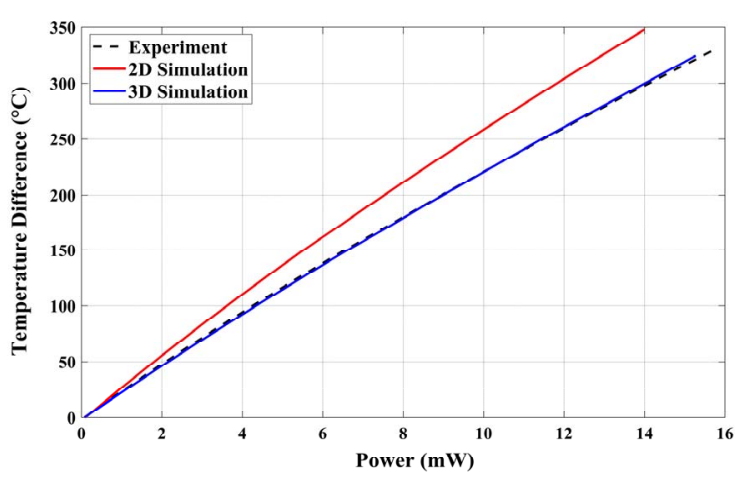

Fig. 5. Temperature reached by the heater for specified power input.

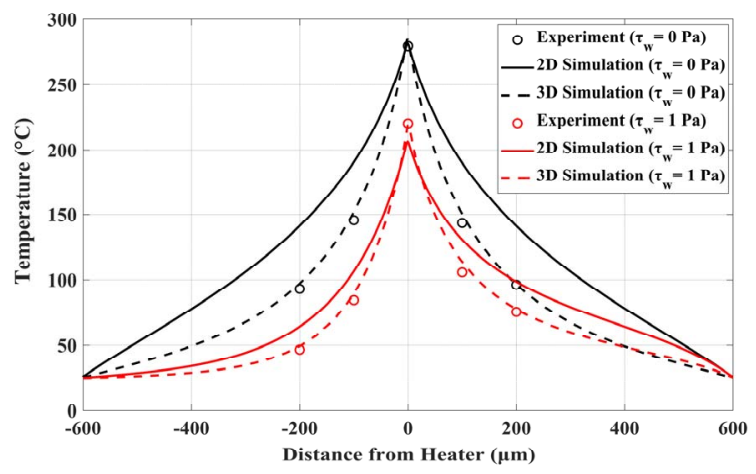

Fig. 6. Temperature profile along the line A-A $\left(\tau_{w}=0\right.$ $\mathrm{Pa})$ and flow $\left(\tau_{w}=1 \mathrm{~Pa}\right)$.

direction. With increasing distance from the heater, the $2 \mathrm{D}$ simulation increases its overestimation due to the omission of the extra dimension for heat dissipation to take place. This error reaches a maximum $(30 \%)$ at the mid-point and then starts to reduce because here the effect of the substrate comes into significance. With the introduction of flow, the same temperature overestimation can be seen.

There are two commonly used methods for thermally measuring the wall shear stress: (a) the anemometric approach (Fig. 7, top), that correlates the temperature difference of the central heater to the incoming flow with respect to the stagnant flow condition, and (b) the calorimetric approach (Fig. 7, bottom), that correlates the temperature difference between two symmetrically situated locations upstream and downstream of the central heater to the incoming flow with respect to the stagnant flow condition. Despite the 3D simulation more closely mapping the on-membrane temperature profile, both 2D and 3D simulations are accurate to within $10 \%$ in predicting the anemometric temperature difference and interestingly, both simulations illustrate the trend produced when 

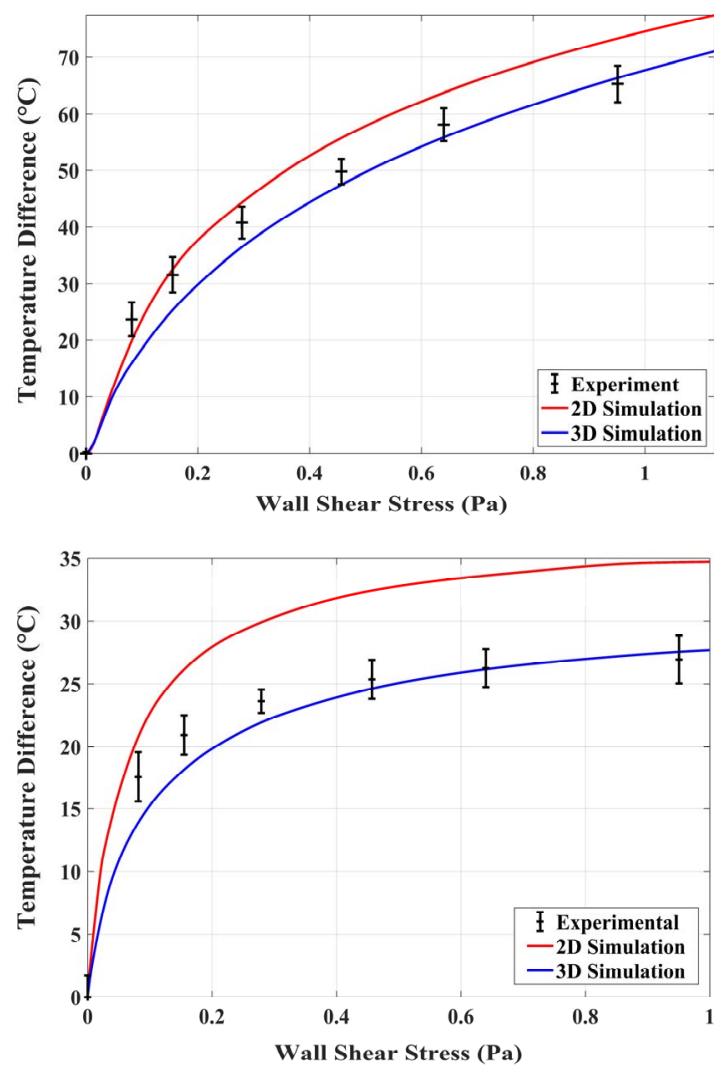

Fig. 7. Temperature difference output as a function of wall shear stress for (top) anemometric approach and (bottom) calorimetric approach at $400 \mu \mathrm{m}$ either side of the heater.

increasing wall shear stress. For the calorimetric approach, the over-estimation of temperature is again present for the 2D simulation. However, it again correctly portrays the trend of increasing wall shear stress, with an initial sharp rise in temperature followed by a gradual decrease in sensitivity. This leads to the conclusion that although $2 \mathrm{D}$ is not as numerically accurate as $3 \mathrm{D}$, it provides sound qualitative results for the behavior of devices.

Table 1 shows the computational resources required to perform the $2 \mathrm{D}$ and $3 \mathrm{D}$ simulations for one set of parameters, i.e. at one flow rate and one bias current. The 2D simulations take less than $2 \%$ of the time required by $3 \mathrm{D}$ leading to a trade-off between time and numerical

Table 1. Computational resources used in performing the numerical modelling (underlined value is an estimation).

\begin{tabular}{|c|c|c|c|}
\hline & $\begin{array}{c}\text { No. of Elements } \\
\text { in Mesh }\end{array}$ & SS Time & $\begin{array}{c}\text { Transient } \\
\text { Time }\end{array}$ \\
\hline 2D & 177,524 & $62 \mathrm{~s}$ & $24 \mathrm{mins} 36 \mathrm{~s}$ \\
\hline 3D & $1,564,734$ & $84 \mathrm{mins} 9 \mathrm{~s}$ & $\underline{33 \mathrm{hrs} 21 \mathrm{mins}}$ \\
\hline
\end{tabular}

accuracy. If detailed quantitative data is essential, 3D simulations must be used, however in order to attain general device behavior, 2D simulations are adequate and extremely time efficient.

It should be noted that all simulations were steady-state (SS) and performed under DC driving mode. However, AC driving modes are often used for thermal flow sensors to reduce the power consumption and to attain information on the thermal properties of the fluid. Transient numerical models use a time marching solution where the defining equations are calculated at every selected time step. This also incorporates many of the higher order terms neglected by steady-state, thus leading to a vast increase in computational resources required. Running the 2D simulation transiently increased the solving time by 24 times, this number was extrapolated to estimate a time for $3 \mathrm{D}$ transient solving. This reveals an unpragmatic simulation and therefore highlights the necessity for $2 \mathrm{D}$ simulations for highly complex problems.

\section{Conclusion}

This paper has presented a comparison between 2D and 3D numerical models for a thermal flow sensor. It is shown, by comparison with experimental data, that 3D models can be used to attain quantitatively accurate data. Whilst providing less accurate data, 2D simulations can still be used to illustrate the behavior and trends of a device with the advantage of demanding less computational resources. This becomes especially prevalent when considering transient simulations.

\section{References}

[1] Q. Lin et al., 'MEMS thermal shear-stress sensors: Experiments, theory and modeling', in Technical Digest of 2000 Solid-State Sensor and Actuator Workshop, pp. 304-307, 2000.

[2] P. Fürjes, G. Légrádi, C. Dücs \Ho, A. Aszódi, and I. Bársony, 'Thermal characterisation of a direction dependent flow sensor', Sens. Actuators Phys., vol. 115, no. 2, pp. 417-423, 2004.

[3] N. Sabaté, J. Santander, L. Fonseca, I. Gràcia, and C. Cané, 'Multi-range silicon micromachined flow sensor', Sens. Actuators Phys., vol. 110, no. 1, pp. 282-288, 2004.

[4] C. Falco, A. De Luca, S. Safaz, I. Haneef, J. Coull, S. Ali, and F. Udrea, '3-D Multiphysics modelling of a SOI CMOS MEMS thermal wall shear stress sensors.' Procedia Engineering, vol. 87, pp. 628-631, 2015.

[5] A. De Luca, C. Falco, E.L.W. Gardner, J.D. Coull and F. Udrea, 'Diode-Based CMOS MEMS Thermal Flow Sensors.' Transducers-2017 Taiwan 20th International Conference on, in press. 